\title{
Regulation of hepatic glucose metabolism by insulin and counter-regulatory hormones
}

\author{
BY KEITH SNELL \\ Receptors and Cellular Regulation Group, School of Biological Sciences, University of Surrey, \\ Guildford GU2 $5 X H$
}

Insulin is the major hormone that regulates glucose homeostasis in the body. It suppresses hepatic glucose production and accelerates glucose utilization in liver and peripheral tissues, resulting in decreased plasma glucose concentration. The regulation of glucose homeostasis is also influenced by counter-regulatory hormones to insulin, such as glucagon, adrenaline, growth hormone or cortisol (Gerich et al. 1980; Cryer, 1981). In the liver, the interaction between these hormones determines the extent of glucose production by glycogenolysis or gluconeogenesis. or both, and the extent of glucose utilization by glycogenesis or lipogenesis, or both. In previously published studies the role of these various counter-regulatory mechanisms and their relationship to insulin action have been examined mainly in short-term situations, such as in response to acute insulin-induced hypoglycaemia. We have sought to define the role of the interplay between insulin and counter-regulatory hormones in controlling hepatic metabolism in situations of chronically altered insulin status. Situations involving chronic hyper- and hypoinsulinaemia have been studied.

\section{GLUCOSE HOMEOSTASIS IN THE NEONATAL RAT AT BIRTH}

The newborn rat (and other mammalian species) is in a transitional state of glucose homeostasis. Whereas the fetus is dependent on the continuous provision of nutrients (in particular, glucose) by the maternal circulation via the placenta, the neonate is subject to a more variable and intermittent exogenous oral intake of nutrients in the form of a low-carbohydrate, high-fat milk diet. The transitional period at birth is a particularly sensitive time since, until suckling begins ( $2-3 \mathrm{~h}$ for the rat), the neonate must depend on the mobilization and oxidation of stored fuels, including the large store of liver glycogen which is synthesized in the final days of gestation. The initiation of hepatic glycogenolysis and gluconeogenesis in the first postnatal hours is critical for the maintenance of glucose homeostasis at this time (Girard et al. 1973; Snell \& Walker, 1973a,b; Snell, 1981). Fetal life is characterized by chronic hyperinsulinaemia which is an important condition for the high rates of fetal growth, and for anabolic pathways of metabolism such as hepatic glycogen deposition. At birth the hyperinsulinaemia continues briefly and is one of the factors involved in the natural delay in hepatic glycogenolysis (Fig. 1(b)) (Snell \& Walker, 1973b, 1978) and functional mitochondrial maturation (Aprille \& Nosek, 1987) which results in a transient postnatal hypoglycaemia in the first few hours of postnatal life (Fig. 1(a)). Counter-regulatory hormone actions are vital for the reversal of the postnatal hypoglycaemia and for establishing glucose homeostasis at this time, but it is not known which hormonal agents are responsible.

It has been assumed that glucagon. presumptively released in response to the postnatal hypoglycaemia, is responsible for initiating glycogenolysis and switching on hepatic 
(b)
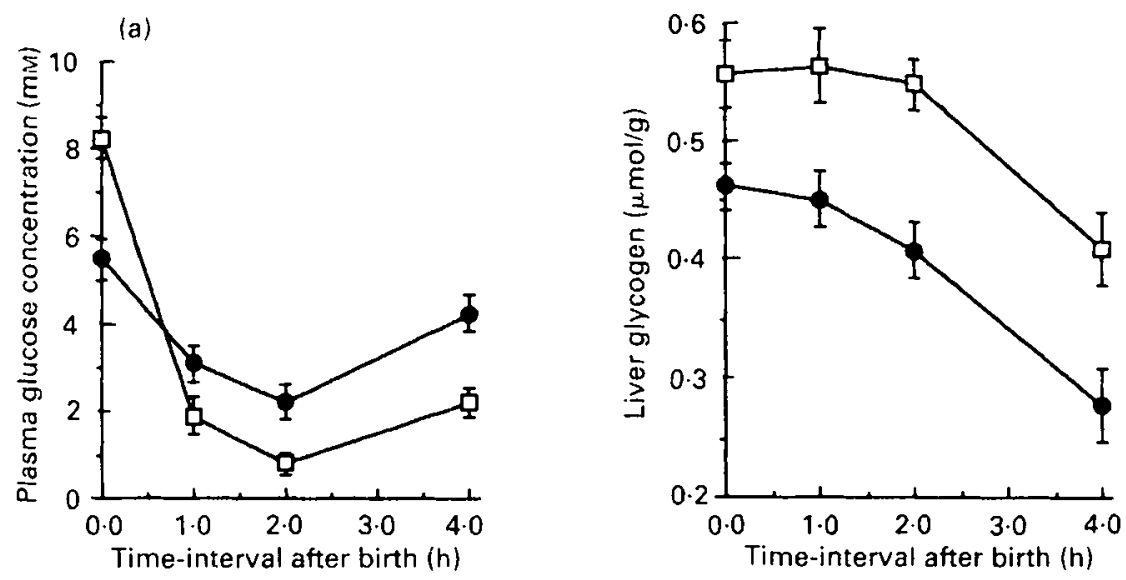

Fig. 1. Plasma glucose concentrations (a) and liver glycogen contents (b) in newhorn rats delivered at term from normal (O) and diabetic $(\square)$ dams. Glucose and glycogen were determined according to Snell \& Walker (1973a). Points are means with their standard crrors, represented by vertical bars. for five to six animals.

gluconeogenesis, and indeed a peak of plasma hormone concentration is observed at 30 min post partum (Girard et al. 1973). However, we have shown in vivo that glucagon is unable to elicit a glycogenolytic response at birth (Snell \& Walker, 1973b, 1978; Snell, 1981). That this is not simply due to the antagonistic interactions prevailing between glucagon and insulin in vivo is shown by our observation that glycogenolysis in neonatal hepatocytes isolated at birth, and incubated in the absence of insulin, is similarly unresponsive to glucagon (Fig. 2). This constitutive lack of glucagon responsiveness is probably due to a decreased concentration (20-30\% of the adult number) and affinity of glucagon receptors in neonatal rat hepatocytes (Pingoud et al. 1982).

It has been suggested that catecholamine secretion at birth may be the critical event in establishing normal glucose homeostasis in the newborn (Sperling et al. 1984). Certainly in the rat, plasma catecholamine concentrations reach a postnatal peak between 1 and $2 \mathrm{~h}$ post partum, coincidental with the nadir in glycaemia and the initiation of measurable hepatic glycogen breakdown (Cuezva \& Patel, 1983). More direct evidence for a glucoregulatory role of catecholamines in the neonatal rat has come from our studics with isolated hepatocytes. In contrast to the unresponsiveness of glycogenolysis to glucagon in these cells, adrenaline is able to stimulate glycogen breakdown and increase hepatocyte glucose production (Fig. 2). The effect of adrenaline is blocked by the $\beta$ - and $\beta$-2-subtype-selective adrenoceptor antagonists (propranolol and ICI 118551), whereas it is only partially blocked by the $\beta$-1-selective adrenoceptor antagonist (atenolol) and not blocked at all by the $\alpha$-selective adrenoceptor antagonist (phentolamine). This establishes that the adrenaline-mediated effect on glycogenolysis in hepatocytes from newborn rats is due to a stimulus-response coupling via the $\beta-2$ adrenergic receptor, and this is in contrast to hepatocytes from adult males which show a predominantly $\alpha$-adrenoceptor-mediated glycogenolytic response (Blair et al. 1979). This is in line with our direct measurements of the ontogenesis of $\beta$-adrenoceptors by radioligand binding assays (Snell \& Evans, 1988). The concentration of total and 


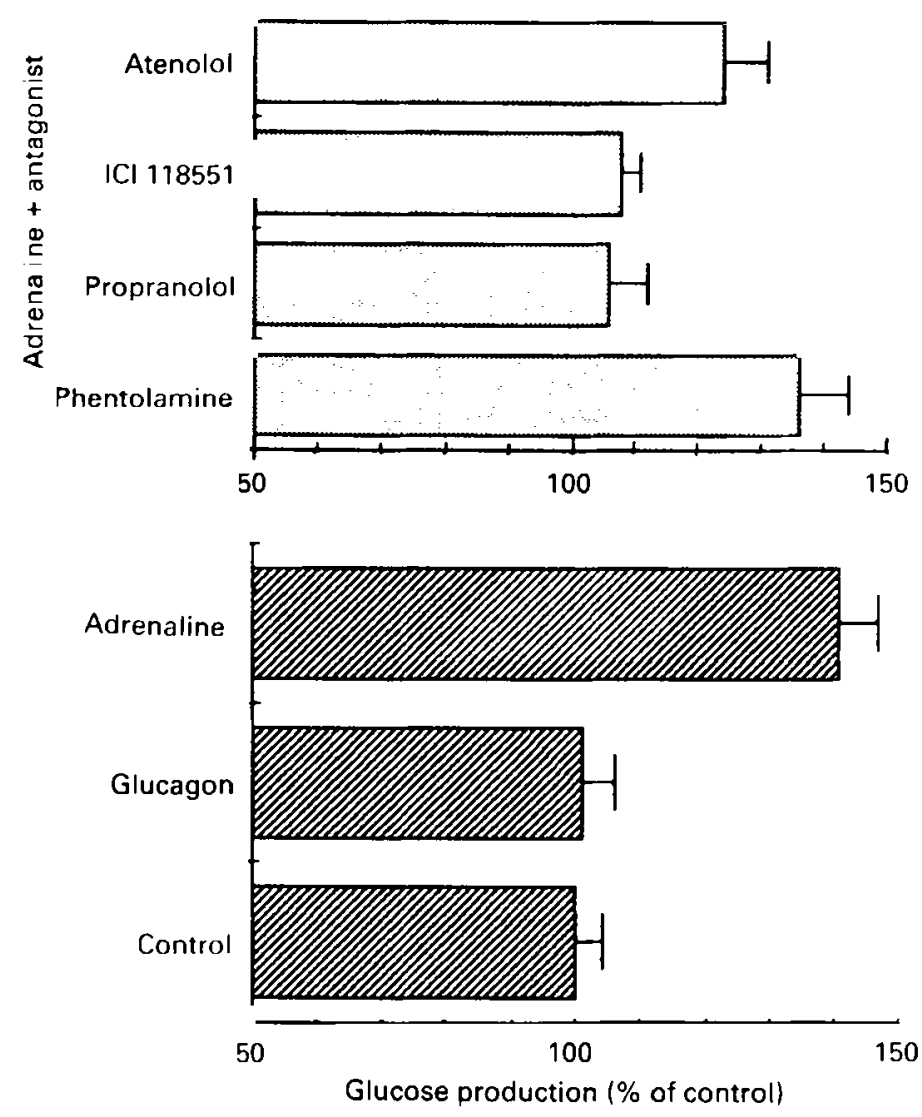

Fig. 2. Glucose production by neonatal hepatocytes in response to glucagon or adrenaline $\left(10^{-6} \mathrm{M}\right)$ or to adrenaline in the presence of $10^{-5} \mathrm{M}$-antagonist. Hepatocytes from term fetal rats were isolated according to Snell \& Evans (1987) and incubated for $30 \mathrm{~min}$ in the absence of additions (control) or additions as indicated. Values are means with their standard errors, represented by vertical bars, for four separate cell preparations.

$\beta$-2-subtype adrenoceptors in hepatic plasma membrane fractions increases during the last days of fetal life to reach a maximum at birth (Fig. 3(a)). In contrast, although $\alpha$-adrenoceptors have been identified in perinatal rat liver by radioligand binding assays, their concentration decreases between late-fetal and early postnatal life (Butlen et al. 1980). Adrenaline-stimulated glucose production by isolated hepatocytes shows a similar ontogenic pattern to that of the $\beta$-2-adrenoceptor, with a peak at birth when the receptor number is at a maximum (Fig. 3(b)). Thus, the evidence from in vitro studies supports the proposal that it is the counter-regulatory actions of the catecholamines that play the critical role in vivo in establishing glucose homeostasis in the newborn rat.

At birth the mobilization of energy stores in the form of hepatic glycogen requires a decrease in plasma insulin levels, and follows the development of hypoglycaemia due to continuing glucose utilization in the absence of either an exogenous or endogenous glucose supply (Snell, 1981). The hypoglycaemia, which is maximal during the second hour after birth, acts as a stimulus for catecholamine secretion which is then able to stimulate hepatic glycogenolysis unopposed by the previously high insulin levels at birth. 

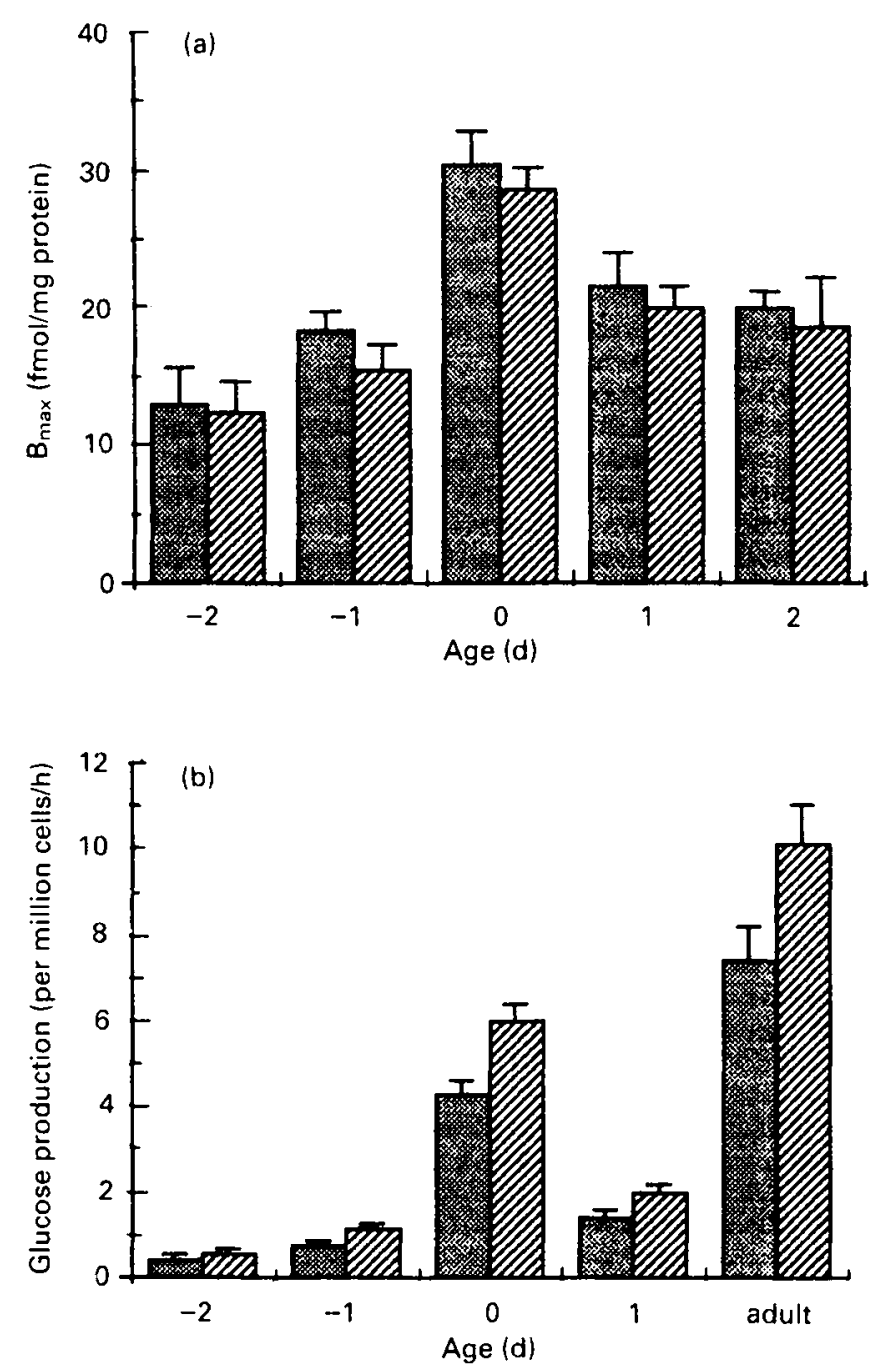

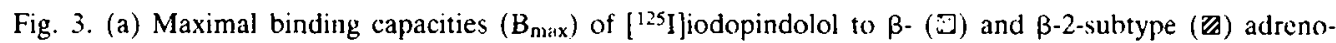
ceptors in liver membrane fractions from perinatal rats of different ages; and (b) glucose production in the absence (包) and presence (0) of $10^{-6} \mathrm{M}$-adrenaline in hepatocytes from perinatal rats of different ages. Radioligand binding assays were carried out according to Snell \& Evans (1988) and perinatal hepatocytes were prepared according to Snell \& Evans (1987). Values are means with their standard crrors, represented by vertical bars, for three to four separate preparations. Age 0 represents term and adult refers to 50 -d-old rats.

Further insight into the interrelationship between insulin and the counter-regulatory actions of catecholamines was obtained by studying hepatic glycogenolytic responses in neonates born to dams made diabetic on day 5 post coitum (by intraperitoneal injection of $45 \mathrm{mg}$ streptozotocin $/ \mathrm{kg}$ body-weight in $50 \mathrm{~mm}$-citrate buffer, $\mathrm{pH} 4.5$ ). The sustained hyperglycaemia of uncontrolled diabetes throughout pregnancy in the dams leads to a chronic fetal hyperglycaemia and consequent hyperinsulinaemia (Cuezva et al. 1982). The elevated plasma insulin in these neonates persists above the levels in normal 
newborn rats for at least the first $6 \mathrm{~h}$ after birth (Cuezva et al. 1982). At birth the neonates of diabetic dams have a higher blood glucose concentration and hepatic glycogen level than is found in normal newborn rats (Fig. 1(a,b)). Despite these augmented glucose reserves, the hyperinsulinaemic neonates experience a more profound and prolonged postnatal hypoglycaemic phase, resulting from an increased delay in mobilizing hepatic glycogen (Fig. $1(a, b)$ ). While in part this may reflect a delayed increase in plasma catecholamine concentrations in these animals (Cuezva et al. 1982), it also involves an intrinsic diminished adrenergic responsiveness of their hepatocytes. Whereas isolated hepatocytes from control newborn rats showed a normal doseresponse relationship to increasing adrenaline concentrations (measuring both glycogen breakdown and glucose production as indices of glycogenolysis), the glycogenolytic response in hepatocytes from hyperinsulinaemic neonates was completely absent, even at $10^{-6} \mathrm{M}$-adrenaline (Fig. 4).

The desensitization of the $\beta$-adrenergic-mediated glycogenolytic response by hyperinsulinaemia in these newborn rats from diabetic dams could involve either receptor or post-receptor mechanisms, or both. Insulin receptor desensitization by $\beta$-adrenergic agonists has been described, and ascribed to a decreased coupling efficiency between insulin binding and receptor kinase activation (Klein et al. 1991), possibly as a result of receptor phosphorylation by cyclic AMP-dependent protein kinase ( $E C$ 2.7.1.37). However, a corresponding $\beta$-adrenergic receptor desensitization by insulin has not been described as yet, although it is well known that insulin acts at a post-receptor level to attenuate cyclic AMP production (e.g. Mor et al. 1981). Regardless of the molecular basis, it is clear that the chronic hyperinsulinacmic state has a profound influence on the expression of the counter-regulatory actions of the adrenergic hormones, with consequent effects on the operation of glucose homeostatic mechanisms. It would be of obvious interest to investigate whether similar effects are operative in other hyperinsulinaemic states, such as obesity or type 2 diabetes (NIDDM).

\section{GLUCOSE HOMEOSTASIS IN THE LACTATING RAT}

In the lactating rat there is an enhanced turnover of glucose imposed by the biosynthetic demands of the mammary gland and the liver in relation to the synthesis of milk constituents (Williamson, 1980; Burnol et al. 1983). In the liver, the increased utilization of glucose is channelled towards fatty acid synthesis, and greater rates of hepatic lipogenesis occur during lactation compared with those in non-lactating animals (Robinson et al. 1978; Williamson, 1980). The increased demands for glucose utilization are met in part by hyperphagia during lactation but, paradoxically, plasma insulin levels are substantially lower in the fed lactating rat compared with the virgin state (Robinson et al. 1978). Thus, although a low circulating insulin concentration may be appropriate for the release of the triacylglycerol-rich lipoprotein from the liver to the mammary gland (Duerden \& Gibbons, 1990), it is seemingly not compatible with the observed increase in flux through the lipogenesis pathway during lactation. This apparent contradiction could be reconciled if there were a diminution in the effectiveness of a counter-regulatory hormone with anti-lipogenic actions during lactation, to an extent which overcame the effects of a lower circulating insulin concentration. Since glucagon and catecholamines are the predominant anti-lipogenic metabolic regulators in mammals, the responsiveness of hepatocytes from lactating rats to these agents was investigated. 

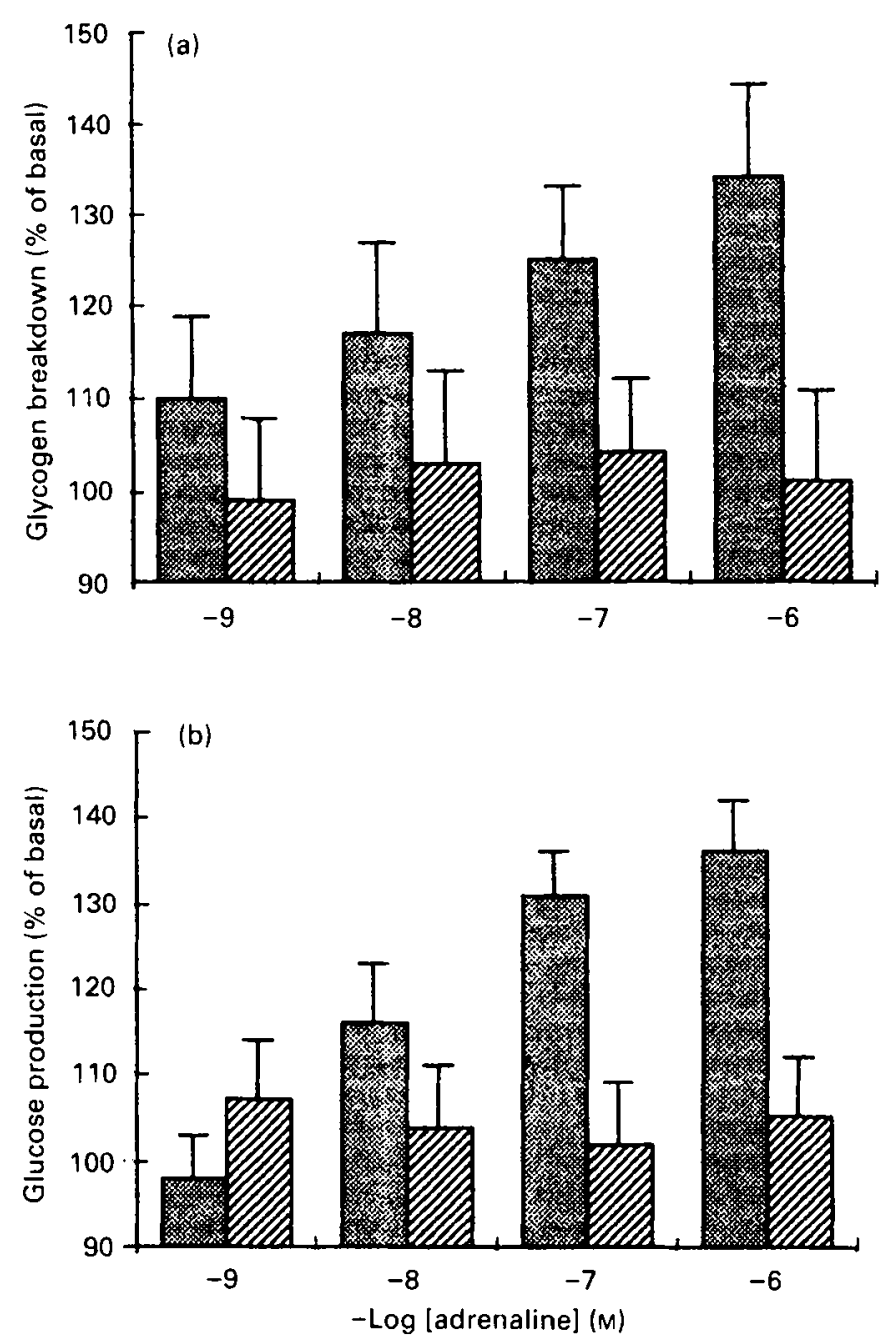

Fig. 4. Glycogen breakdown (a) and glucose production rates (b) in hepatocytes from newborn rats from normal (国) and diabetic (dams at different concentrations of adrenaline. Measurements of glycogen and accumulation of glucose (as $\mu \mathrm{mol} / 10^{6}$ cells per $\mathrm{h}$ ) were made in hepatocytes incubated for $30 \mathrm{~min}$ with adrenaline at the concentrations indicated. Values are expressed as a percentage of the basal rate in the absence of adrenaline. Hepatocytes were prepared according to Snell \& Evans (1987). Values are means with their standard errors, represented by vertical bars, for four separate hepatocyte preparations.

Using a radioligand binding assay with [ ${ }^{125}$ I]iodopindolol and liver membrane preparations (Snell \& Evans, 1988) we have found no difference in receptor number or affinity of $\beta$-adrenoceptors in lactating rats compared with non-lactating animals (Dunphy et al. 1989). Similar determinations of $\alpha$-1-adrenoceptors using $\left[{ }^{3} \mathrm{H}\right]$ prazosin have also shown no difference in this receptor subtype in lactation (A. M. Dunphy, R. A. Clegg and K. Snell, unpublished results). Post-receptor events were evaluated in hepatocytes by measuring the adenylate cyclase (EC 4.C.1.1)-mediated increase in cyclic 

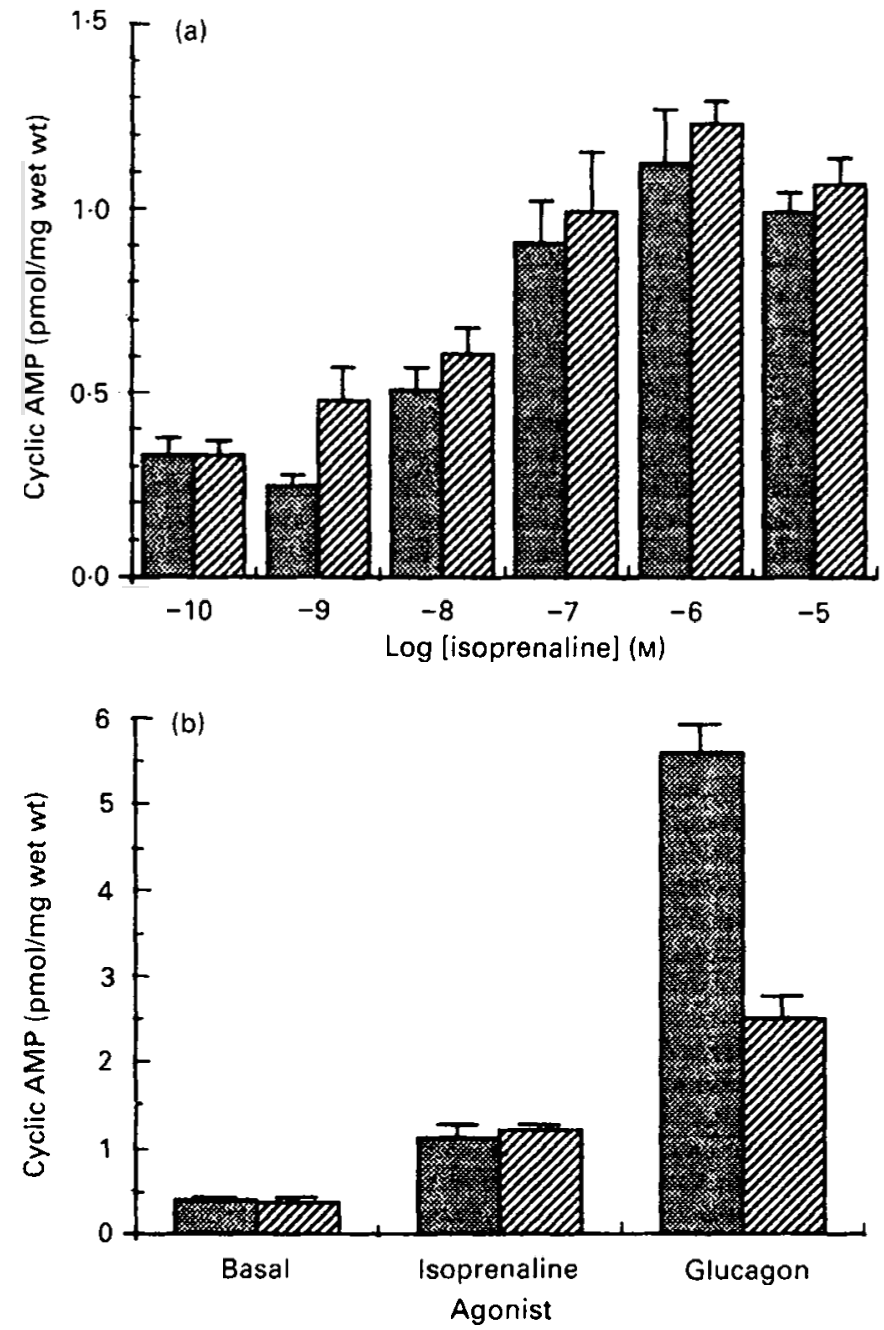

Fig. 5. Stimulation of cyclic AMP accumulation in hepatocytes from lactating ( $(2)$ and non-lactating (国) rats (a) at different concentrations of isoprenaline and (b) at $10^{-6} \mathrm{M}$-isoprenaline or glucagon or under unstimulated basal conditions. Cyclic AMP content was measured in hepatocytes after a $60 \mathrm{~s}$ incubation period according to Clegg \& Mullaney (1985). Values are means with their standard errors, represented by vertical bars. for six separate hepatocyte preparations.

AMP levels in response to the $\beta$-adrenergic agonist isoprenaline (Fig. 5(a)). Again, no difference was observed in the dose-response relationship between hepatocytes from lactating compared with non-lactating female rats. However, when the effects of maximally stimulating doses of isoprenaline and of glucagon (both $10^{-6} \mathrm{M}$ ) were compared, glucagon was a much more effective activator of adenylate cyclase than the $\beta$-adrenergic agonist (Fig. 5(b)). More significantly, there was a markedly diminished activation of adenylate cyclase by glucagon in hepatocytes from lactating rats compared with non-lactating animals. 
The maximal unstimulated activity of adenylate cyclase in hepatocytes was not affected by lactational status and, since isoprenaline-stimulated activity was also not affected, the diminished responsiveness of cyclic AMP production to glucagon must be due to a reduction in the number of glucagon receptors or to an attenuation of receptor coupling to adenylate cyclase. The factor(s) responsible for the desensitization of the glucagon response remain to be identified, but it seems that in the hypoinsulinaemic lactating rat this may result in a diminished opposition by glucagon of the effects of insulin on hepatic metabolism. In other words, this could explain the observed utilization of glucose for an increased rate of lipogenesis in lactating rat liver despite the prevailing hypoinsulinaemia which otherwise might mitigate against the biosynthetic metabolism. This raises the possibility that the modulation of the counter-regulatory hormonal response, rather than changes in insulin itself, may play the predominant role in certain circumstances in controlling glucose homeostasis.

\section{CONCLUSIONS}

The control of glucose homeostasis by metabolic actions directed at the liver are clearly under the influence of insulin. However, in both the physiological examples considered previously, and involving hyper- and hypoinsulinaemic states, there is an essential and additional dependence on modulations of counter-regulatory hormonal responses to effectively control glucose homeostasis. The counter-regulatory hormones involved are different in the two situations examined (catecholamines in hepatic glycogenolysis at birth, and glucagon in hepatic lipogenesis during maternal lactation), but in both cases there is the suggestion that the modulation of hormonal responsiveness occurs at the level of the hormone receptor itself or its signal transduction coupling mechanism.

These studies were carried out in collaboration with Dr Roger A. Clegg, Andrea M. Dunphy, Dr Carole A. Evans, Dr Sam Kacew and Professor Deryck G. Walker, and with the financial support of Glaxo Group Research Ltd, the Hannah Research Institute, the Science and Engineering Research Council and the Wellcome Trust.

\section{REFERENCES}

Aprille, J. R. \& Nosek, M. T. (1987). Neonatal hypoxia or maternal diabetes delays postnatal development of liver mitochondria. Pediatric Research 21, 266-269.

Blair, J. B., James, M. E. \& Foster, J. L. (1979). Adrenergic control of glucose output and adenosine 3',5'-monophosphate levels in hepatocytes from juvenile and adult rats. Journal of Biological Chemistry 254, 7579-7584.

Burnol. A. F. Leturque. A., Ferré, P. \& Girard. J. (1983). Glucose metabolism during lactation in the rat: quantitative and regulatory aspects. American Journal of Physiology 245, 7579-7584.

Butlen, D., Guillon, C., Cantau, B. \& Jard, S. (1980). Comparison of the developmental patterns of vasopressin, glucagon and $\alpha$-adrenergic receptors from rat liver membranes. Molecular Cell Endocrinology 19, $275-289$.

Clegg, R. A. \& Mullaney, 1. (1985). Acute changes in the cyclic AMP content of rat mammary acini in vitro. Biochemical Journal 230, 239-246.

Cryer, P. E. (1981). Glucose counterregulation in man. Diabetes 30, 261-264.

Cuezva, J. M., Burkett, E. S., Kerr, D. S., Rodman, H. \& Patel, M. S. (1982). The newborn of diabetic rat. 1. Hormonal and metabolic changes in the postnatal period. Pediatric Research 16, 632-637.

Cuezva, J. M. \& Patel, M. S. (1983). Plasma catecholamine concentrations in the newborn rat during the first six postnatal hours. Biochemical Society Transactions 10, 521 . 
Duerden, J. M. \& Gibbons, G. F. (1990). Storage, mobilization and secretion of cytosolic triacylglycerol in hepatocyte cultures. The role of insulin. Biochemical Journal 272, 583-588.

Dunphy, A. M.. Clegg, R. A. \& Snell, K. (1989). Characterisation of rat liver $\beta$-adrenoceptors during lactation. Biochemical Society Transactions 17, 1098-1099.

Gerich, J.. Cryer. P. E. \& Rizza, R. (1980). Hormonal mechanisms in acute glucose counterregulation: the relative roles of glucagon. epinephrinc, norepinephrine. growth hormone and cortisol. Metabolism 29. $1164-1175$.

Girard, J. R., Cuendet, G. S., Marliss, E. B., Kervran, A., Rieutort, M. \& Assan, R. (1973). Fuels, hormones, and liver metabolism at term and during the early postnatal period in the rat. Journal of Clinical Investigation 52, 3190-3200.

Klein. H. H., Matthaei, S., Drenkhan, M., Ries, W. \& Scriba, P. C. (1991). The relationship between insulin binding. insulin activation of insulin-receptor tyrosine kinase, and insulin stimulation of glucose uptake in isolated rat adipocytes. Biochemical Journal 274, 787-792.

Mor, M. A., Vila, J., Ciudad, C. J. \& Guinovart, J. J. (1981). Insulin inactivation of rat hepatocyte cyclic AMP-dependent protein kinase. FEBS Letters 136, 131-134.

Pingoud, V. A., Petcrs, F., Haas, T. D. U. \& Trautschold, I. (1982). A quantitative analysis of glucagon binding to isolated intact neonatal and adult rat hepatocytes on the basis of two different binding models. Biochimica et Biophysica Aclu 714, 448-455.

Robinson, A. M.. Girard, J. R. \& Williamson, D. H. (1978). Evidence for a role of insulin in the regulation of lipogenesis in lactating rat mammary gland. Biochemical Joumal 176, 343-346.

Snell, K. (1981). Glucose turnover in the newborn rat. In Metabolic Adaptation to Extrauterine Life. pp. 81-105 [R. de Meyer, editor]. The Hague: Martinus Nijhoff Publishers.

Snell, K. \& Evans, C. A. (1987). Isolation of fetal and neonatal rat hepatocytes. In Biochemical Toxicology: A Practical Approach, pp. 80-83 [K. Snell and B. Mullock, editors]. Oxford: IRL Press.

Snell, K. \& Evans, C. A. (1988). Characterization of rat liver $\beta$-adrenoceptors during perinatal development as determined by [12.51]-iodopindolol radioligand binding assays. British Journal of Pharmacology 93, 817-826.

Snell, K. \& Walker, D. G. (1973a). Glucose metabolism in the newborn rat: temporal studies in vivo. Biochemical Journal 132, 739-752.

Sncll, K. \& Walker, D. G. (1973b). Glucose metabolism in the newborn rat: hormonal cffects in vivo. Biochemical Journal 134. 899-906.

Sncll. K. \& Walker. D. G. (1978). Glucose metabolism in the newborn rat: the role of insulin. Diabetologia 14, $59-64$.

Spcrling, M. A., Ganguli. S.. Leslie, N. \& Landt, K. (1984). Fetal-perinatal catecholamine secretion: role in perinatal glucose homeostasis. American Journal of Physiology 247, E69-E74.

Williamson, D. H. (1980). Integration of metabolism in tissues of the lactating rat. FEBS Letlers 117, K93-K105. 